\title{
Los recursos forestales maderables y el desarrollo social y económico en el estado de Durango
}

\author{
Gustavo Pérez Verdín ${ }^{1}$
}

\begin{abstract}
RESUMEN
En este estudio se hizo un análisis comparativo de las condiciones sociales y económicas de los municipios con recursos forestales maderables, aquellos sin recursos forestales maderables y aquellos sensitivos a potenciales cambios en la política forestal. El análisis de las condiciones económicas y sociales que caracterizan a los habitantes de las áreas geográficas forestales constituye una base imprescindible para la programación socioeconómica, tanto en el ámbito del sector público como del privado. Por ejemplo, la asignación de recursos de inversión, para fines de desarrollo general o para la expansión empresarial, requiere del conocimiento sobre las particularidades de las circunscripciones territoriales. El estudio se desarrolló en el estado de Durango y se basó en la compilación de 51 variables de tipo social y económico incluyendo el grado de marginación y la migración trasnacional y su relación con el estado actual de los recursos forestales maderables, caracterizados predominantemente por bosques de clima templado con asociaciones de pino, encino y otras coníferas. Anualmente, la producción forestal maderable en el estado es de 1,8 millones de $\mathrm{m}^{3} \mathrm{r}$, lo que lo coloca como el principal productor forestal del país, y su valor económico asciende a alrededor de \$120 MD EUA. Con todo y sus riquezas naturales, Durango es el séptimo estado del país expulsor de emigrantes hacia los Estados Unidos de América y el décimo sexto con el índice de marginación más alto en el país. El estudio recomienda diversificar el uso de los recursos naturales y dar prioridad a aquellos municipios sensitivos al momento de hacer la asignación de recursos económicos.
\end{abstract}

PALABRAS CLAVE:

Comunidades sensitivas, dependencia de recursos, desarrollo socioeconómico, recursos forestales maderables.

\section{ABSTRACT}

This paper analyzed some socio-economic conditions of forest-dependent, non-forest dependent, and policy-sensitive municipalities in the state of Durango, Mexico. Knowledge of the socioeconomic relationships among variables that determine the main characteristics of a geographic area is important for planners and decision makers in both public and private sectors. For example, assigning monetary resources for socio-economic development or business expansion requires recognition of the territory boundaries in which the projects are embedded. The study used 51 variables to assess the socio-economic conditions, including poverty and migration indexes, and its influence on current forest conditions. Durango's forests are characterized by pine, oak, and other conifer associations. Yearly, the forest production hits 1,8 million $\mathrm{m}^{3} \mathrm{r}$ and generates around USD $\$ 120$ million. While this figures position the state as the first forest producer, it ranks seventh among the Mexican states with the highest rate of illegal immigration, primarily to the USA, and sixteenth with the highest poverty index. The study recommends diversify the use of natural resources and prioritize those policy-sensitive counties when planning and assigning monetary resources.

KEY WORDS:

Policy-sensitive communities, forest-dependency, socio-economic development, wood resources. 


\section{INTRODUCCIÓN}

La economía en México está polarizada, con la concentración de la riqueza en las ciudades principales, especialmente en la capital del país o en las ciudades capitales de los estados (Hernández-Díaz, 1988). Esta centralización provoca la emigración de la población de las áreas rurales a las áreas urbanas, incluso hacia los EUA, además de desempleo y marginación. Los efectos de esta polarización se manifiestan aun cuando muchos de los recursos y oportunidades de desarrollo existen en las áreas rurales y a pesar de los esfuerzos del gobierno federal y estatal para promover incentivos y fuentes de empleo en las áreas rurales, especialmente en aquellas con un desarrollo socioeconómico notablemente bajo.

La literatura reporta que la gente que vive en áreas dependientes de los recursos naturales son pobres y se encuentran en desventaja con otras áreas que no dependen de los recursos naturales (Humphrey et al., 1993). La dependencia en recursos naturales es una realidad que se conceptualiza basándose en teorías de sistemas abiertos donde los individuos intercambian recursos con el medio ambiente como una condición para sobrevivir (USDA Forest Service ,1999). La necesidad de adquirir los recursos necesarios para la subsistencia de estas comunidades crea dependencia y puede causar problemas que requieren soluciones de tipo político. El papel de los administradores es manejar los recursos sin crear dependencias difíciles y que pongan en peligro la integridad de los ecosistemas.

En México y en otras partes del mundo, muchos municipios se caracterizan no solo por tener una fuerte dependencia con los recursos forestales, sino también por un bajo desarrollo social y económico, como insuficiente infraestruc- tura caminera en la región, desempleo, falta de escuelas, hospitales, etc. Eventualmente, estos factores ocasionan otros fenómenos sociales como migración, inseguridad y destrucción de los recursos naturales, etc. El estado de Durango cuenta con muchas riquezas naturales, por ejemplo es el primer estado en producción forestal con un total de 1,8 millones de $\mathrm{m}^{3} \mathrm{r}$ anuales y una derrama económica de alrededor de 120 MD EUA (INEGI, 2003). Es fuente reguladora de múltiples servicios ambientales como agua, belleza escénica, recreación, secuestro de carbón, biodiversidad, etc. Además, los recursos forestales dan empleo directo a más de 18,000 personas. Sin embargo, el estado de Durango es el séptimo expulsor de emigrantes principalmente hacia Estados Unidos y el décimo sexto con mayor índice de marginación (CONAPO, 1997).

En este estudio se pretendió identificar y cuantificar las condiciones sociales y económicas de municipios dependientes del recurso forestal y contribuir en el conocimiento de algunos índices socioeconómicos, como el Índice de Marginación, índice de Intensidad Migratoria y el Índice de Diversidad Económica en municipios del estado de Durango que dependen de los recursos forestales maderables y en aquellos que no dependen de este tipo de recursos. La diferenciación de áreas sensitivas a posibles cambios en la política de la administración de los recursos forestales maderables (por ejemplo una reducción en los volúmenes de corta anual, para proteger una especie determinada o para favorecer ciertos servicios ambientales) es fundamental para propósitos de asignación de recursos económicos federales o estatales o para simplemente evaluar el potencial riesgo de expandir un negocio o proyecto regional. En estos casos, es necesario determinar la unidad física de manejo o el territorio para identificar $y$ 
cuantificar las variables necesarias que simulen el comportamiento de las condiciones sociales y económicas y su relación con los recursos naturales.

\section{OBJETIVOS}

Los objetivos del presente trabajo de investigación fueron: identificar las variables requeridas para hacer un análisis integral de las condiciones sociales y económicas a nivel municipal en el estado de Durango y su relación con la situación actual de los recursos forestales maderables. A manera de hipótesis, el estudio determinará si los municipios con recursos forestales maderables cuentan con un menor desarrollo económico que los que no dependen de los recursos forestales maderables. Además, identificará los municipios sensitivos a cualquier cambio en la política forestal y determinará si estos se encuentran aun más rezagados que los municipios menos sensitivos a ese tipo de cambios políticos o administrativos.

\section{METODOLOGÍA}

\section{Área de estudio}

El estado de Durango se localiza en el noroeste del país y cuenta con una superficie territorial de poco más de 12 millones de hectáreas. El sistema montañoso de la Sierra Madre Occidental divide al estado en dos secciones generando la "región de las montañas", donde se localiza el bosque templado y pequeñas porciones de selva subhúmeda, y la "región de valles" que incluye la zona del semidesierto y matorrales (Fig. 1). La entidad comprende 39 municipios; tres de ellos se localizan en ambientes urbanos y el resto se localiza en el medio rural. A nivel nacional, el estado de Durango cuenta con la reserva de bosques más grande del país (aproximadamente 5 millones de ha) los cuales proveen múltiples bienes y servicios que no son aprovechados y distribuidos eficientemente. Por ejemplo, la mayoría de los municipios

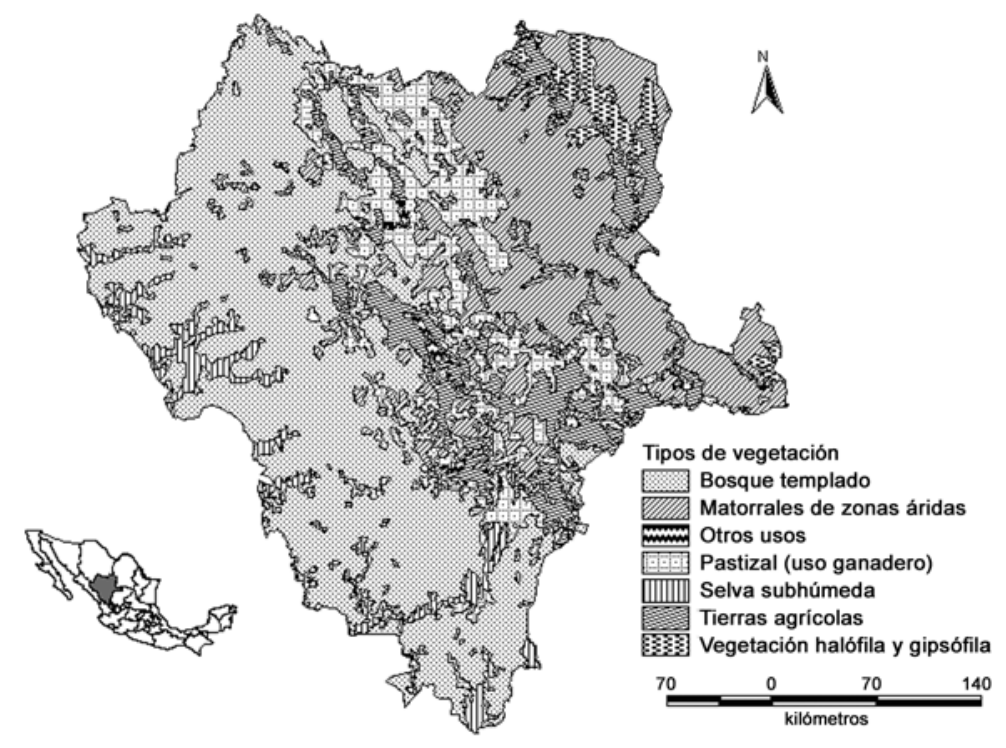

Figura 1. Localización del estado de Durango y el área con recursos forestales maderables (bosque templado) 
forestales dependen de la producción maderable como la principal actividad económica del sector forestal $y$, salvo casos esporádicos, se deja de lado la oferta de otros servicios ambientales, como el agua, recreación / ecoturismo, protección del suelo y biodiversidad.

Este trabajo se basó en el municipio como la unidad geográfica mínima de comparación. El municipio es la instancia de gobierno más cercana a la población y al territorio local. Sin embargo, debido al centralismo del Estado, ha sido una de las instancias más débiles. Dicha debilidad se ha traducido en la incapacidad real para enfrentar los complejos problemas de su jurisdicción y en su papel subordinado hacia los niveles federal y estatal (Ávila-García, 1997). Su función básicamente se ha centrado en proporcionar los servicios urbanos de la población que se asienta sobre todo en las cabeceras municipales.

No obstante, el municipio es la unidad de medida más real para establecer parámetros de comparación y definir propuestas de planeación. Algunas de las razones que favorecen esta decisión son: Primero, existe una adecuada base de datos a esta escala, colectada consistentemente por el Instituto Nacional de Estadística, Geografía e Informática (INEGI, 2003), la cual es usada para los censos estadísticos nacionales. Segundo, el municipio es la unidad de planeación para distribuir los presupuestos gubernamentales federales y/o estatales. Programas como el Ramo 33 que comprende beneficios económicos a los municipios de la entidad, y el Programa de Empleo Temporal se basan fundamentalmente en la distribución política de los municipios. Tercero, el municipio es la unidad sociopolítica más cercana al manejo de ecosistemas. Las ciudades y pueblos, por ejemplo, son demasiado pequeños y los estados incluyen muchos y variados tipos de ecosistemas (Force et al., 1997). Por lo tanto, la información a nivel municipal es una estrategia eficaz para evaluar las condiciones socioeconómicas en las áreas forestales del estado de Durango.

La base de información de este estudio provino de distintas instituciones de recopilación de información e investigación del gobierno federal, estatal y municipal, además de otras fuentes e investigadores particulares en la materia. Se generaron 51 variables para cada uno de los 39 municipios de la entidad y se construyeron tres índices socioeconómicos.

Para clasificar los municipios dependientes de los recursos forestales maderables, se utilizó el porcentaje de superficie cubierta con recursos forestales maderables y el porcentaje de empleos generados por la actividad forestal. Se consideraron municipios forestales maderables aquellos que tuvieron más del $40 \%$ de territorio cubierto con bosques y que más del $10 \%$ de empleos fueron generados por el sector forestal. La excepción fue el municipio de Durango, el cual debido a su tamaño y heterogeneidad fue incluido dentro de los municipios no forestales. De esta manera se identificaron 13 municipios con recursos forestales maderables y 26 sin recursos forestales maderables (Tabla 1). Análisis de varianza estadística de una vía y pruebas de $t$ fueron usados para diferenciar los dos grupos de municipios con base en los índices socioeconómicos y el conjunto de variables analizadas en el estudio.

Además, dentro de los municipios con recursos forestales maderables se identificaron aquellos más sensitivos a potenciales cambios en la administración de los recursos naturales o cambios por otros factores económicos. Por ejemplo, ¿cuál sería el efecto de una reducción en los aprovechamientos forestales si una 
Tabla 1. Clasificación de los municipios del estado de Durango, de acuerdo a la existencia de recursos forestales maderables

\begin{tabular}{ccc}
\hline $\begin{array}{c}\text { MUNICIPIOS CON RECURSOS } \\
\text { FORESTALES MADERABLES }\end{array}$ & MUNICIPIOS SIN RECURSOS FORESTALES MADERABLES \\
\hline Canelas & Canatlán & Panuco de Coronado \\
Guanaceví & Coneto & Peñón Blanco \\
Mezquital & Cuencamé & Poanas \\
Nuevo Ideal & Durango & Rodeo \\
Ocampo & El Oro & San Juan de Gpe. \\
Otaéz & Gómez Palacio & San Juan del Río \\
Pueblo Nuevo & Guadalupe Victoria & San Luis del Cordero \\
San Bernardo & Hidalgo & San Pedro del Gallo \\
San Dimas & Inde & Santa Clara \\
Santiago Papasquiaro & Lerdo & Simón Bolívar \\
Tamazula & Mapimí & Suchil \\
Tepehuanes & Nazas & Vicente Guerrero \\
Topia & Nomblilo de Dios &
\end{tabular}

mayor superficie forestal se dejara para otorgar servicios ambientales a la sociedad en general?, ¿cuáles serían los municipios más sensibles a esta reducción de volúmenes de corta? o ¿a cuáles municipios se les debería considerar prioritarios en términos de asignación de presupuesto, apoyos económicos de instancias federales, estatales 0 no gubernamentales? El análisis de los municipios sensitivos se basó en variables como el Índice de Diversidad Económica, el Índice de Marginación, sueldo per capita, la importancia de la actividad forestal en el PIB municipal, la proporción del número de empleos forestales y la proporción del área forestal (Cook y Mizer, 1994).

\section{Selección de variables}

Existen muchas variables e indicadores que contribuyen a la evaluación de las condiciones sociales y económicas en un área (Force et al., 1997). En este caso, la selección de las variables e indicadores estuvo basada en los siguientes criterios: (1) revisión de literatura; (2) relevancia de la variable para correlacionar el desarrollo social y económico con los aprovechamientos forestales; (3) facilidad para ser entendidos y aplicados; (4) disponibilidad de datos; y (5) accesibilidad de buenas fuentes.

La tabla 2 presenta las variables e indicadores utilizados en el análisis. 
Tabla 2. Variables socioeconómicas utilizadas en el análisis de los municipios de Durango

\begin{tabular}{|c|c|c|}
\hline CRITERIO & INDICADOR & ESCALA DE INDICADOR \\
\hline Recursos naturales & $\begin{array}{l}\text { Bosques } \\
\text { Volumen de aprovechamientos } \\
\text { forestales }\end{array}$ & $\begin{array}{l}\text { ha } \\
\mathrm{m}^{3} \text { rollo por año }\end{array}$ \\
\hline Grado de urbanización & $\begin{array}{l}\text { Agua potable } \\
\text { Electricidad } \\
\text { Drenaje } \\
\text { Baños }\end{array}$ & $\begin{array}{l}\% \text { de viviendas } \\
\% \text { de viviendas } \\
\% \text { de viviendas } \\
\% \text { de viviendas }\end{array}$ \\
\hline Infraestructura & $\begin{array}{l}\text { Hoteles } \\
\text { Escuelas } \\
\text { Aeropuertos / Pistas de aterrizaje } \\
\text { Hospitales } \\
\text { Servicio postal } \\
\text { Industria } 1 \\
\text { Densidad de caminos } \\
\text { Caminos pavimentados }\end{array}$ & $\begin{array}{l}\text { Número } \\
\text { Número } \\
\text { Número } \\
\text { Número } \\
\text { Número } \\
\text { Número de industrias } \\
\mathrm{km} / \mathrm{ha} \\
\mathrm{km}\end{array}$ \\
\hline Aspectos demográficos & $\begin{array}{l}\text { Población total } \\
\text { Densidad de población } \\
\text { Crecimiento de población } \\
\text { Índice de marginación } 2 \\
\text { Índice de intensidad } \\
\text { migratoria } 2 \text { (USA) } \\
\text { Alfabetismo } \\
\text { Educación superior }\end{array}$ & $\begin{array}{l}\text { Habitantes } \\
\text { Habitantes / } \mathrm{km}^{2} \\
\% \text { de cambio } \\
\text { No aplica } \\
\text { No aplica } \\
\% \text { de población alfabeta } \\
\% \text { de población con } \\
\text { educación superior }\end{array}$ \\
\hline $\begin{array}{l}\text { Aspectos } \\
\text { socioeconómicos }\end{array}$ & $\begin{array}{l}\text { Producción total } \\
\text { Salarios promedio mensuales } \\
\text { Valor de la producción maderable } \\
\text { Empleos }{ }^{3}\end{array}$ & $\begin{array}{l}\text { Miles \$ / año } \\
\text { Miles \$ / persona } \\
\text { Miles \$ / año } \\
\text { Número de empleos }\end{array}$ \\
\hline
\end{tabular}

1 La información se dividió en las principales clases de fuentes de empleo según el Censo Económico 1999 del INEGI: manufactura, pesca, minería, comercio, transportación, servicios y construcción.

2 Los índices de marginación e intensidad migratoria fueron calculados por la conAPO utilizando técnicas de componentes principales.

3 En el concepto de empleos se utilizó el Índice de Diversidad Económica (Shannon y Weaver) para calificar cada uno de los municipios de manera homogénea. Las clases de empleo se subdividieron en: (1) Agricultura, ganadería; (2) Minería; (3) Extracción de petróleo; (4) Industria manufacturera; (5) Electricidad y agua; (6) Construcción; (7) Comercio; (8) Transportes y comunicaciones; (9) Servicios financieros; (10) Administración pública y defensa; (11) Servicios comunales y sociales; (12) Servicios profesionales y técnicos; (13) Servicios de hoteles y restaurantes; (14) Servicios personales y mantenimiento; (15) Otros. 
Para reducir el número de variables de la tabla anterior se utilizó la técnica de Análisis de Componentes Principales con matriz de correlaciones, el cual es un método matemático que transforma un conjunto de variables (o indicadores) en uno nuevo, donde con un número menor de variables se pretende reelaborar una interpretación más sencilla del panorama original (Tabachnick y Fidell, 2001). Una vez que fueron detectados los principales componentes en la matriz de correlaciones, se utilizaron los coeficientes de predicción para crear una nueva variable en función del número de componentes encontrados. Esto es, si el análisis determinó la existencia de tres componentes principales, se crearon tres nuevas variables que, a su vez, representaron al conjunto de variables en cada componente principal. Posteriormente, los componentes principales fueron diferenciados entre sí tomando como referencia los municipios con recursos forestales maderables y sin recursos forestales maderables utilizando pruebas estadísticas de $t$ con un nivel de significancia $(\alpha)$ de 0,05 . El procesamiento de la información se desarrolló mediante el paquete estadístico SPSS versión 13,0.

Índices de comparación

Con base en información de INEGI, SEMARNAT, CONAFOR, CONAPO, UJED, $y$ Gobierno del Estado de Durango, se obtuvieron y determinaron los siguientes índices socioeconómicos:

1. Índices de marginación, que se basan en variables de tipo social y demográfico. Los índices de marginación son indicadores de la calidad de vida en las distintas regiones del país. Bajos índices de marginación indican que la calidad de vida es mejor y viceversa. En Durango, los municipios con índices de marginación más altos se localizan precisamente en la región donde se encuentran los recursos forestales maderables y en la región del semidesierto. El índice de marginación fue desarrollado por la cONAPO (http://www. conapo.gob.mx/00cifras/ marg2000/ anexo_b.XLS, último acceso, 13 de septiembre de 2005) tomando en cuenta las siguientes variables: Porcentaje de población analfabeta; porcentaje sin primaria completa; porcentaje de viviendas sin drenaje, electricidad, agua entubada, piso de tierra, hacinamiento; porcentaje de población en localidades menores de 5,000 habitantes; y porcentaje de población con ingresos de hasta dos salarios mínimos.

2. Índices de diversidad económica por municipio, basado en el Índice de Diversidad de Shannon y Weaver (Horne y Haynes, 1999):

$$
D=-1 \sum_{i=1}^{n}\left(E_{i} * \log E_{i}\right)
$$

donde $D$ es el índice de diversidad y $E$ es la proporción de empleo total en cada municipio. El índice de diversidad económica es una herramienta eficaz para determinar el nivel de resiliencia que tiene una comunidad a potenciales cambios en la administración pública o al efecto de otros fenómenos macroeconómicos (Hornes y Haynes, 1999). Índices altos indican que la comunidad es más resiliente a potenciales cambios en la administración pública y que los habitantes tendrían mayores oportunidades de empleo en la región. En este caso, 
para construir este índice se utilizaron datos de empleo provenientes de las principales fuentes de empleo en la región, incluyendo agricultura, ganadería, silvicultura, pesca, minería, petróleo, industria manufacturera, electricidad, servicios financieros, comercio, transportación, servicios de restaurante y hoteles, y construcción (Tabla 2). Los municipios duranguenses con mayor diversidad económica son los que se encuentran en ambientes urbanos, esto es Durango, Gómez Palacio y Lerdo.

3. Índices de Intensidad Migratoria, los cuales fueron originalmente desarrollados por la CONAPO para diferenciar entidades y municipios según la intensidad global del fenómeno migratorio a estas escalas (http://www.conapo.gob.mx/publicaciones/migra4.htm, último acceso, 13 de septiembre de 2005). Para calcular este índice, la cONAPO utilizó cuatro variables básicas: Hogares que reciben remesas, hogares con emigrantes en EUA del quinquenio anterior, hogares con migrantes circulares del quinquenio anterior y hogares con migrantes de retorno.

Los tres índices se utilizaron para caracterizar a cada uno de los municipios y hacer también las comparaciones entre los municipios con recursos forestales maderables y sin recursos forestales maderables. La razón de incluir estos indicadores se debe a la agrupación genérica de variables que involucró cada uno de ellos y a la importancia que tienen en el desarrollo socioeconómico de los municipios. Las potenciales diferencias estadísticas entre los dos tipos de municipios fueron evaluadas mediante la prueba $t$.

\section{RESULTADOS}

\section{Reducción de variables}

El Análisis de Componentes Principales detectó tres componentes principales (factores). El $79,2 \%$ del total de la varianza fue atribuible a estos tres componentes y el procedimiento fue estadísticamente significativo con un valor de $p<0,001$ (Bartlett's test of Sphericity). La tabla 3 muestra los valores de los coeficientes de correlación entre las variables utilizadas y cada uno de los componentes principales detectados. En esta tabla puede observarse que en el componente 1 , las variables con mayor coeficiente de correlación fueron escuelas, hospitales, servicio postal, hoteles, industrias y población total. El segundo componente estuvo caracterizado por el Índice de Marginación, electricidad, drenaje, baños, agua entubada y alfabetismo. El tercer componente por densidad de caminos, densidad de población y producción total.

Con base en las variables representativas de cada componente, se denominó al primer componente como Infraestructura, al segundo componente como vivienda y al tercer componente como poblacional. Una vez que fueron identificados los tres componentes, se procedió a estimar, por regresión, los valores de los municipios en cada uno de los tres componentes creados. Se utilizó la prueba estadística $t$, para comparar si existen diferencias entre los municipios con recursos forestales maderables y sin recursos forestales maderables. En la tabla 4 se aprecia que las variables representadas por el componente 2: vivienda, tuvieron diferencias significativas entre los municipios con recursos forestales maderables y $\sin$ recursos forestales maderables. Los primeros tuvieron valores negativos en la componente de vivienda. Esto indica que, en general, los recursos forestales made- 
rables han contribuido a mejorar por igual la infraestructura de los municipios. Sin embargo, existen diferencias significativas a nivel familiar, esto es en términos de electricidad, drenaje, baños, agua entubada y alfabetismo, lo que significa que los recursos forestales maderables no han contribuido a mejorar las condiciones de vida de los habitantes de los municipios forestales de la misma manera que aquellos habitantes de los municipios sin recursos forestales.

Tabla 3. Matriz de Componentes Principales y coeficientes de correlación utilizando el método Varimax de rotación

\begin{tabular}{lccc}
\hline VARIABLES & \multicolumn{3}{c}{ COMPONENTES PRINCIPALES } \\
& \multicolumn{2}{c}{3} \\
\hline Escuelas & 0,961 & 0,004 & 0,214 \\
Hospitales & 0,945 & 0,115 & 0,108 \\
Servicio Postal & 0,932 & 0,275 & 0,004 \\
Hoteles & 0,922 & 0,269 & $-0,026$ \\
Industrias & 0,895 & 0,295 & 0,243 \\
Población total & 0,890 & 0,255 & 0,325 \\
Educación superior & 0,663 & $.0,521$ & 0,392 \\
Sueldo anual & 0,603 & $-0,188$ & 0,305 \\
Pistas de aterrizaje & 0,565 & $-0,409$ & $-0,205$ \\
Caminos pavimentados & 0,533 & 0,497 & 0,206 \\
Índice de Intensidad Migratoria & $-0,394$ & 0,233 & $-0,261$ \\
Índice de Marginación & $-0,152$ & $-0,946$ & $-0,167$ \\
Electricidad & $-0,101$ & 0,933 & 0,025 \\
Drenaje & 0,152 & 0,886 & 0,137 \\
Baños & 0,092 & 0,878 & 0,122 \\
Agua entubada & 0,147 & 0,867 & 0,131 \\
Alfabetismo & $-0,008$ & 0,843 & 0,062 \\
Índice de Diversidad Económica & 0,291 & 0,548 & 0,113 \\
Densidad de población & 0,247 & 0,200 & 0,936 \\
Densidad de caminos & 0,053 & 0,284 & 0,933 \\
Producción total & 0,599 & 0,207 & 0,739 \\
& & & \\
\hline
\end{tabular}

Tabla 4. Promedio de los valores de regresión para las componentes principales por tipo de municipio

\begin{tabular}{lccc}
\hline COMPONENTE & $\begin{array}{c}\text { MUNICIPIOS SIN RECURSOS } \\
\text { FORESTALES MADERABLES }\end{array}$ & $\begin{array}{c}\text { MUNICIPIOS CON } \\
\text { RECURSOS FORESTALES } \\
\text { MADERABLES }\end{array}$ & VALOR DE P \\
\hline 1 Infraestructura & $-0,072$ & 0,145 & 0,53 \\
2 Vivienda & 0,484 & $-0,968$ & 0,00 \\
3 Poblacional & 0,038 & $-0,076$ & 0,74 \\
\hline
\end{tabular}


Entre las causas que, aparentemente, han motivado los rezagos en las condiciones de vida de los habitantes de municipios con recursos forestales se encuentra que estos se localizan en zonas poco accesibles y remotas, lo cual hace difícil llevar los servicios necesarios a toda la población; la alta dispersión de poblaciones menores de 100 habitantes, (http://www.inegi.gob.mx/est/contenidos/e spanol/rutinas/ept.asp? $\mathrm{t}=\mathrm{mpob} 96 \& \mathrm{c}=384$ $1 \& \mathrm{e}=10$, último acceso, 13 de sep. 2005); la poca atención a los municipios con grupos indígenas (alfabetismo); a una baja diversificación del uso del bosque para fines no maderables (recreación, ecoturismo, producción de agua, etc.) y, en consecuencia, una ineficiente asignación de los recursos económicos, traducida en bajos presupuestos para el desarrollo de recursos no maderables y/o servicios ambientales (Pérez-Verdín y Tecle, 2004).

\section{Comparación de índices}

Con el fin de entender las relaciones entre los Índices de Marginación, Intensidad Migratoria y Diversidad Económica, se realizó un análisis bivariado de correla- ción entre ellos. El Índice de Marginación tuvo una correlación negativa con el Índice de Diversidad Económica (-0,59, $p<0,001)$, pero no se encontró correlación alguna con el Índice de Intensidad Migratoria. Tampoco se encontró relación alguna entre el Índice de Diversidad Económica y el de Intensidad Migratoria. En otras palabras, no existe relación alguna entre los niveles de marginación y migración, pero a medida que la marginación aumenta, el Índice de Diversidad Económica decrece. Aparentemente, el fenómeno de migración en Durango se da por factores no relacionados con la falta de empleo, sin embargo, habría que explorar otras causas que favorecen la migración como son: la falta de empleos bien remunerados, cultura (o tradiciones familiares de migrar a los EUA) y/o falta de información.

Para diferenciar estadísticamente estos índices entre los municipios con recursos forestales maderables y sin recursos forestales maderables se aplicó una prueba de $t$ para evaluar sus diferencias. La figura 2 muestra los resultados de estos análisis, en donde se observan diferencias significativas en los tres índices y los tipos de municipios.

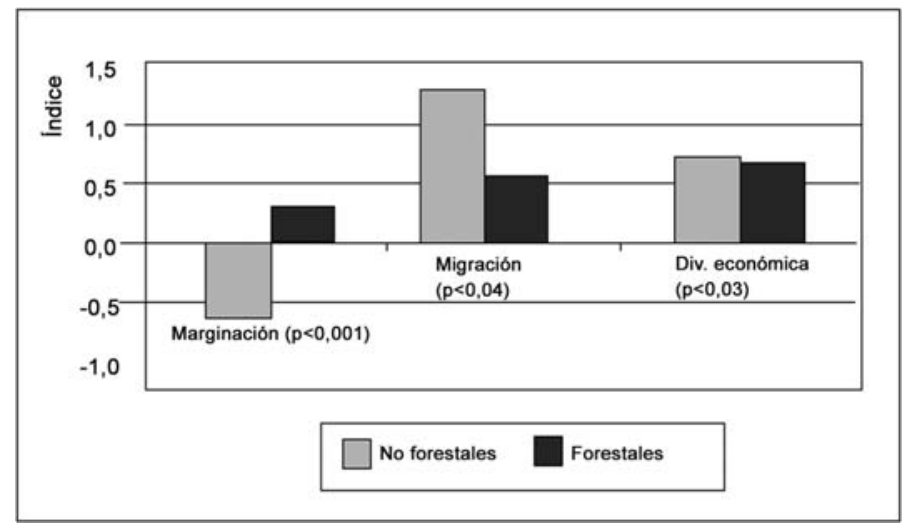

Figura 2. Representación de los índices de marginación, migración y diversidad económica. Por ser índice, no hay una unidad de medida representativa en el eje $y$. El nivel de significancia $(p)$ fue determinado $\alpha=0.05$ 
El índice de marginación es más alto en los municipios con recursos forestales maderables, situación que se puso de manifiesto en el análisis de las variables a nivel vivienda. La tasa de migración es más alta en los municipios sin recursos forestales maderables, debido quizás, al mayor número de habitantes. Es importante notar que, los municipios con el mayor índice de migración se localizan en la zona del semidesierto. El Índice de Diversidad Económica es ligeramente mayor en los municipios sin recursos forestales maderables, debido también a la mayor población que habita en los municipios sin recursos forestales.
Municipios sensitivos. Con base en los datos de índices de marginación, diversidad económica, sueldo per capita, porcentaje de la producción forestal con respecto a la producción total (como medida del PIB forestal a nivel municipal), proporción de empleos generados por la actividad forestal y el porcentaje de área cubierta con recursos forestales maderables, se determinó que los municipios de Canelas y Tamazula presentan los mayores índices de sensibilidad a potenciales cambios en la administración y manejo de los recursos forestales maderables. Los municipios con menor sensibilidad y más resilientes fueron Santiago Papasquiaro y Nuevo Ideal (Fig. 3).

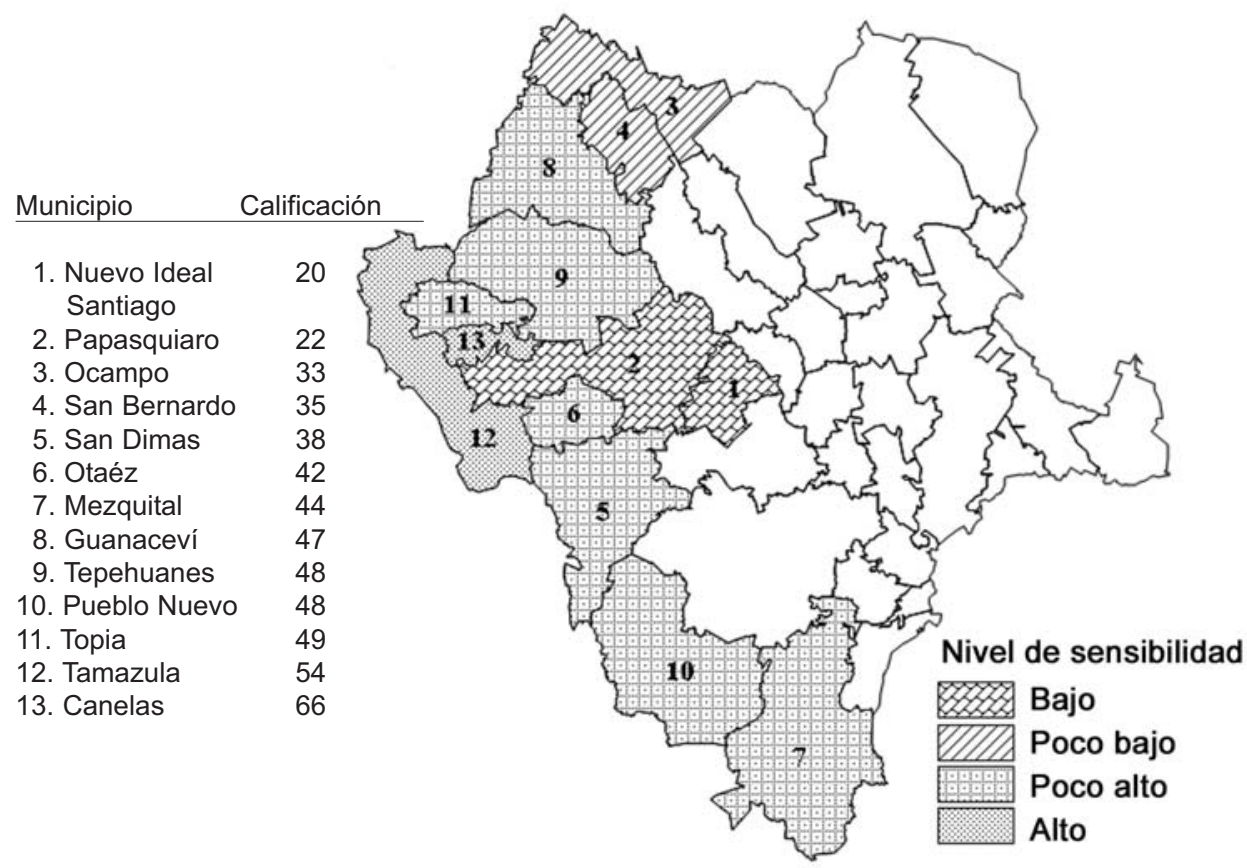

Figura 3. Municipios sensitivos a cambios potenciales en la administración y manejo de los recursos forestales maderables. El nivel de sensibilidad está basado en: índice de marginación, sueldo promedio, diversidad económica, área forestal, empleos y PIB forestal 


\section{DISCUSIÓN Y CONCLUSIONES}

Si bien es cierto que el presente estudio se basó en el análisis de variables que involucran a una región específica del país, éste sugiere que el desarrollo integral de los municipios con recursos forestales maderables, en general, está íntimamente ligado al manejo de dichos recursos. El estudio no encontró diferencias significativas en cuanto a la infraestructura de los dos tipos de municipio, pero sí a nivel hogar o vivienda. En este caso, los municipios que dependen de los recursos forestales maderables se encuentran más rezagados en materia de desarrollo socioeconómico y en desventaja con otros municipios que no dependen de los recursos forestales maderables. El estudio sugiere, también, que la teoría de dependencia de recursos forestales puede ser aplicable a muchas áreas forestales del país. Los resultados coinciden con los trabajos de Humphrey et al. (1993) al determinar que la gente que vive en áreas dependientes de los recursos naturales son pobres y se encuentran en desventaja con otras áreas que no dependen de los recursos naturales.

Con el propósito de reducir las diferencias entre el nivel socioeconómico de los municipios con y sin recursos forestales maderables es importante que:

1. Los servicios ambientales (agua, suelo, belleza escénica, ecoturismo, etc.) que se producen en los municipios forestales sean revaluados para que los pobladores tengan fuentes alternativas de ingresos y motivación para la conservación de los recursos. Este estudio determinó diferencias significativas en cuanto a la diversidad económica en los dos tipos de municipios. Algunas de las razones que explican esas diferencias son que los municipios con recursos forestales maderables dependen básicamente de la producción maderera. Una mayor diversificación significaría mayor resiliencia y diversidad económica.

2. Los apoyos directos para las comunidades y los recursos forestales maderables deben ser distribuidos en forma equitativa, tomando en consideración a los municipios más sensitivos o altamente dependientes de los recursos forestales maderables. De la misma manera, la asignación del presupuesto federal/estatal del sector forestal debe basarse en proyectos con alto impacto en los servicios ambientales (Pérez-Verdín y Tecle, 2004).

3. Se recomienda más investigación en la evaluación del índice de marginación (pobreza) y su potencial relación con la deforestación de los bosques.

\section{RECONOCIMIENTOS}

Este estudio fue desarrollado con financiamiento de México-North Research Network, Inc. y Northern Arizona University. Fue parcialmente presentado durante la reunión binacional entre México y Estados Unidos en las instalaciones de la Secretaría de Relaciones Exteriores, México, D.F., en julio del 2003. El autor agradece los comentarios y revisiones de los doctores Denver Hospodarsky y J. Ciro Hernández-Díaz de Northern Arizona University e Instituto de Silvicultura e Industria de la Madera-UJED, respectivamente; así como los valiosos comentarios de dos revisores anónimos.

\section{REFERENCIAS}

Ávila-García, P. 1997. El municipio y la gestión ambiental del desarrollo local. In: G. López Castro (Editor) 
Sociedad y Medio Ambiente en México. México: El Colegio de Michoacán, A.C. p. 54-73.

CONAPO. 1997. Síntesis del estudio binacional México-EUA sobre Migración. Boletín, Migración Internacional, 1 (4): nov-dic. México, D.F.

Cook, P.J. y K.L. Mizer. 1994. The revised ERS county typology: an overview. Rural Development Res. Rep. No. 89. Washington, DC: U.S. Department of Agriculture, Economic Research Service. 48 p.

Force, J., E. Machlis, y E. Gary. 1997. The human ecosystem part II: Social Indicators in Ecosystem Management. Society and Natural Resources 10 (4): 369-377

Hernández-Díaz, J.C. 1988. Input-Output analysis of the state of Durango, Mexico. [Ph D. Dissertation] Colorado State University. Fort Collins, CO. 157 p.

Horne, A.L. y R.W. Haynes. 1999. Developing measures of socioeconomic resiliency in the Interior Columbia Basin. PNW-GTR-453. Pacific Northwest Research Station. USDA Forest Service. Portland, OR. 41 p.

Humphrey, C.R., G. Berardi, M.S. Carrol et al. 1993. Theories in the study of natural resource-dependent communities and persistent rural poverty in the U.S. In: Rural Sociological Society Task Force on Persistent Rural Poverty (Editor). Persistent Rural Poverty in America. Boulder, CO: Westview Press. p. 133-173

INEGI. 2003. Anuario Estadístico de Durango. México, INEGI. (Accessible en http://www.inegi.gob.mx/ prod_serv/contenidos/espanol/ biblioteca/ default.asp?accion= \&upc $=702825158415 \&$ seccionB $B=b$, última visita 13 de sep. 2005).

Pérez-Verdín, G. y A. Tecle. 2004. Use of the Analytic Hierarchy Process in forest budget allocation in Durango, México. Hydrology and Water Resources in Arizona and the Southwest 34: 39-49.

Tabachnick, B.G. y L.S. Fidell. 2001. Using multivariate statistics. Needham Heights, MA: Allyn \& Bacon. 966 p.

USDA Forest Service. 1999. OzarkOuachita highlands assessment: Social and economic conditions. Report 4 of 5. GTR SRS-34. North Central Research Station and Southern Research Station. Asheville, NC. 299 p. 
